\title{
Impact of Priming on Effectiveness of TMS in Detecting Language-eloquent Brain Areas in Tumor Patients
}

\author{
Sascha Freigang ${ }^{1,2}$ Shane Fresnoza ${ }^{3,4}$ Kariem Mahdy Ali ${ }^{1}$ Karla Zaar $^{1}$ Margit Jehna ${ }^{5}$ \\ Gernot Reishofer ${ }^{5}$ Katrin Rammel ${ }^{1} \quad$ Fritz Studencnik $^{5}$ Anja Ischebeck ${ }^{3,4}$ Gord von Campe ${ }^{1}$
}

${ }^{1}$ Department of Neurosurgery, Medical University of Graz, Graz,
Austria
${ }^{2}$ Department of Basic and Clinical Neuroscience, King's College
London, London, United Kingdom
${ }^{3}$ Institute of Psychology, University of Graz, Graz, Austria
${ }^{4}$ BioTechMed, Graz, Graz, Austria
${ }^{5}$ Department of Radiology, Medical University of Graz, Graz, Austria

Address for correspondence Sascha Freigang, MD, Department of Neurosurgery, Medical University of Graz, Graz 8010, Austria (e-mail: saschafreigang@gmail.com).

J Neurol Surg A 2020;81:111-129.

\begin{abstract}
Keywords

- language mapping

- repetitive transcranial magnetic stimulation

- direct cortical stimulation

- tumor patient

- plasticity

Background and Study Aims Language is characteristically human, and preserving it is critical when resecting tumors in language-eloquent brain areas. Navigated repetitive transcranial magnetic stimulation (nrTMS) has been used in recent years as a noninvasive technique to identify preoperatively the language-eloquent cortical areas in tumor patients. An important objective is to increase the sensitivity and specificity of nrTMS in detecting language-related areas and increase the positive correlation of its results to that of intraoperative direct cortical stimulation (DCS). Although the technical aspects of the procedure have received enormous interest, factors related to the targeted cortical area such as previous cortical history or activity have been neglected. Therefore, the present study explores the impact of previous cortical history or activity on the effectiveness of a subsequent nrTMS mapping paradigm.

Materials and Methods Twelve right-handed patients with a left hemispheric glioma underwent presurgical nrTMS language mapping and intraoperative language mapping with DCS. nrTMS was performed using a continuous theta burst stimulation paradigm to inhibit possible language relevant areas in the vicinity of the tumor, determined anatomically or based on functional magnetic resonance imaging hotspots. The nrTMS was applied in two separate sessions. One of the sessions randomly included a priming paradigm to precondition the targeted cortical areas.

Results Priming stimulation decreased the error detection of the subsequent nrTMS mapping paradigm. This effect was more robust on major types of errors such as speech arrest and hesitation.

Conclusion Prior cortical activity as induced by the priming stimulation has a profound impact on the responsiveness to the nrTMS mapping paradigm. Our findings further showed that metaplasticity, a type of homeostatic plastic process, could be elicited even in cortical areas affected by a growing tumor.
\end{abstract}

received

December 18, 2018

accepted after revision

April 16, 2019

published online

January 14, 2020 (c) 2020 Georg Thieme Verlag KG

Stuttgart · New York
DOI https://doi.org/

10.1055/s-0039-1698382.

ISSN 2193-6315. 


\section{Introduction}

Language processing is considered a uniquely human ability that ought to be preserved in tumor surgery. The resection of tumors located in language-eloquent brain areas, however, bears a high risk of surgery-related language impairment. At present, functional magnetic resonance imaging (fMRI) and direct cortical stimulation (DCS) during awake craniotomy are the routine pre- and intraoperative language mapping procedures. ${ }^{1,2}$ However, this multimodal approach suffers from several constraints, particularly fMRI during preoperative planning. Intracerebral lesions induce edema, change oxygenation, increase cerebral blood volume, and invade normal brain parenchyma affecting the blood-oxygenlevel-dependent (BOLD) signal. ${ }^{3}$ These changes, in addition to the brain shift during surgery, limit the intraoperative use of preoperative fMRI data. ${ }^{4,5}$ This could explain why language mapping obtained with naming or verb generation tasks during fMRI does not perfectly correlate with intraoperative brain mapping results. ${ }^{6}$ Additionally, the limited test-retest reliability and high variability of the fMRI signal is another problematic factor, especially for clinical studies with a small sample size. ${ }^{7}$

Therefore, it is not surprising that a review of nine brain mapping studies concluded that due to inconsistent and contradictory results, fMRI cannot be considered an alternative mapping tool to DCS for tumors located in languageeloquent areas. ${ }^{8}$ In the last few years, several centers incorporated the use of a noninvasive brain stimulation technique called repetitive transcranial magnetic stimulation (rTMS) in combination with a neuronavigation system (navigated rTMS or nrTMS) to further improve presurgical language mapping in tumor patients. ${ }^{9,10}$ Clinical benefits include a possible reduction of required craniotomy size and operative time, as well as preserved language ability after surgery. $3,10,11$

TMS involves the application of magnetic pulses over the intact scalp inducing a secondary electric field in the underlying cortex that in turn can elicit a physiologic response like neuronal spiking. ${ }^{12}$ In the motor cortex, the repetitive application of single magnetic pulses (rTMS) with identical interstimulus intervals at low $(\geq 1 \mathrm{~Hz})$ and high $(\geq 5 \mathrm{~Hz})$ frequencies can suppress or increase neuronal excitability, respectively. ${ }^{13-15} \mathrm{~A}$ similar effect can be achieved by delivering three to five pulses in a burst with different interstimulus intervals at theta rhythm: continuous theta burst stimulation (cTBS) has an inhibitory effect, whereas intermittent TBS (iTBS) has a facilitatory effect, on motor cortex excitability. ${ }^{16}$ The effects of inhibitory rTMS on the brain are long-term depression (LTD)-like and that of excitatory rTMS are long-term potentiation (LTP)-like, because the duration of the aftereffects seem to implicate changes in synaptic plasticity. ${ }^{17}$ Inhibitory rTMS paradigms resemble DCS because both can induce a transient "virtual lesion" that inhibits cortical excitability. ${ }^{18,19}$ For instance, inhibitory rTMS to the left inferior and prefrontal cortex disrupted speech production (particularly for verbs), as well as semantic judgments. ${ }^{20-22}$ Conversely, excitatory paradigms applied to the left frontal cortex areas enhanced syntactic processing and improved verb production. ${ }^{23,24}$ Therefore, it is possible to identify specifically the cortical regions responsive to rTMS that presumably are language relevant. ${ }^{1}$

Over the years, research has refined the preoperative TMS language mapping protocols and increased their reliability. ${ }^{9}$ So far, the focus has been on the technical aspects of the procedure including the addition of a frameless stereotactic navigation system with the TMS coil and pulse generator, allowing precise real-time navigation and quantification of the magnetic field. The inclusion of video and audio recording allows offline data analysis. Optimization of stimulation frequency, intensity, coil orientation, and pulse-train timing maximizes the sensitivity and specificity of the method. ${ }^{3,9,25-29}$ The preoperative identification of the patient's language-dominant hemisphere using rTMS helped predict permanent aphasia after surgery. ${ }^{30}$

However, no study has yet explored the influence of factors related to the state of the cortical areas being stimulated that could modulate the aftereffects of the rTMS stimulation during language mapping in tumor patients. One of these factors is the "cortical history," the state of the cortical circuits before functional mapping. Early studies suggested that the direction of the stimulation aftereffect (LTP versus LTD) or the degree of synaptic plasticity (short versus long lasting) induced by a stimulation paradigm can be influenced by the prior synaptic or cellular activity of the targeted brain area. ${ }^{31}$ This synaptic property is called "plasticity of synaptic plasticity," or metaplasticity.

This property means that the effect of the stimulation cannot be predicted unless the previous stimulation history of the tissue is known. ${ }^{31,32}$ Experimentally, metaplasticity can be probed through the manipulation of the target areas' initial activation state by preconditioning ("priming") it with the same or a different stimulation paradigm. ${ }^{33,34}$ For instance, pretreatment of the motor cortex by high-frequency $(6 \mathrm{~Hz})$ stimulation increases the ability of subsequent $1-\mathrm{Hz}$ stimulation to induce in vitro LTD. ${ }^{35}$ In the language domain, priming 1-Hz stimulation of Wernicke's area with rTMS at the same frequency facilitated the detection of native words, whereas priming 1-Hz stimulation of Wernicke's area with cTBS facilitated the detection of foreign words. ${ }^{34}$ Furthermore, an improvement was reported for expressive and receptive language abilities in poststroke aphasia patients who underwent 6-Hz primed low-frequency rTMS combined with intensive speech therapy. ${ }^{36}$

At present, it is unknown if the phenomenon of metaplasticity holds true for language-associated areas in the vicinity of the tumor and to what extent it can affect the results of functional language mapping. In principle, a highly variable interindividual neuronal state (e.g., altered baseline cortical excitability) mediated by different tumor grades, variable tumor sizes, the extent of infiltration, premorbid language reorganization (compensatory functional plasticity), and the use of different drugs may alter a patient's sensitivity to the stimulation. It might modify the ability of the stimulation paradigm to induce subsequent synaptic plasticity such as LTP or LTD. ${ }^{32}$ We examined this issue by 
comparing two language mapping protocols differing mainly in the presence or absence of a cTBS priming paradigm before the functional mapping procedure. We hypothesized that preconditioning the cortical area to be tested with a cTBS priming paradigm will boost the inhibitory effect of the stimulation during language mapping and improve the detection of true language-positive areas. Additionally, interindividual variability should decrease because the priming paradigm will set a uniform baseline state before the real stimulation. As a secondary objective, we will correlate the results of both primed and unprimed TMS stimulation with the intraoperative DCS findings.

\section{Materials and Methods}

\section{Patients}

Twelve patients (six men) with a mean age of $42.75 \pm 4.27$ years (mean \pm standard error of the mean [SEM]) were included in the study. They all had tumors located in the vicinity or within the perisylvian regions in the left brain hemisphere and were scheduled for awake craniotomy. No patients had motor deficits, and they all had normal or corrected-to-normal vision. All were right-handed according to the Edinburgh Handedness Inventory. ${ }^{37}$ Inclusion criteria were the absence of aphasia, as well as any contraindications to TMS $^{38}$ Exclusion criteria included the history of other neurologic/psychologic/psychiatric disorders (especially epilepsy [not associated with the tumor], schizophrenia, mania, or depression), head injury with loss of consciousness, intracerebral ischemia/history of cerebral hemorrhage as well as serious medical conditions (e.g., cardiovascular disease). Further exclusion criteria were alcohol or drug addiction, pregnancy or breastfeeding, intake of any central nervous system active drugs (e.g., anticonvulsants) at least 2 to 3 hours before rTMS.

Patients were only included if there were no legal reasons why they could not participate and they had not participated in another scientific or clinical study within the last 8 weeks. All patients were enrolled in the study at least 2 weeks before their scheduled tumor resection. The experimental set-up was in accordance with the guidelines set by the Declaration of
Helsinki and approved by the Ethics Committee of the Medical University of Graz (reference number 28-144 ex 15/16). All patients signed written informed consent before the study.

\section{Study Design}

The study was conducted in a single-blinded randomized design. Each patient underwent one preoperative fMRI session and two preoperative randomized nrTMS sessions (with and without priming) separated by an interval of at least 1 week to avoid carryover effects (-Fig. 1). Tumor resection was performed at least 1 day or 3 days after the unprimed and primed TMS session, respectively.

\section{Preoperative fMRI Language Mapping}

The study always began with an fMRI language mapping session performed on a 3-T clinical scanner with a 32channel head coil (Prisma fit, Siemens Healthineers, Erlangen, Germany). The protocol included the acquisition of an isovolumetric T1-weighted, three-dimensional (3D) data set using a magnetization-prepared rapid gradient-echo (MPRAGE) sequence (TR/TE, 1,900/2.2 ms; TI, $900 \mathrm{ms;} \mathrm{flip}$ angle, 9 degrees; 176 sagittal slices; matrix size, $256 \times 256 \mathrm{~mm}^{2}$; voxel size, $1 \times 1 \times 1 \mathrm{~mm}^{3}$; and acquisition time, 3:25 min). The scanning protocol also included the acquisition of BOLD T2*-weighted functional images using a single-shot gradient-echo echo-planar imaging sequence (TR/TE, 2,500/35 ms; flip angle, 90 degrees; slice thickness, $3 \mathrm{~mm}$; resolution, $3 \times 3 \times 3.6 \mathrm{~mm}$; matrix size, $64 \times 64$; field of view, $192 \mathrm{~mm}$; 41 slices per volume; distance factor, 25\%; and GRAPPA, 2). During fMRI, all subjects performed a silent object-naming (naming on visual picture presentation) task, a silent sentence generation (sentence generation on visual noun presentation) task, and simple motor tasks (e.g., finger tapping) to evaluate movements of the fingers, toes, and tongue. They were instructed to perform the task at a selfpaced rate. The paradigms were chosen for their easy implementation and high interindividual reproducibility.

After the procedure, the imaging data were processed and analyzed with the help of the FEAT tool from the free FSL software packages (https://fsl.fmrib.ox.ac.uk/fsl/fslwiki, FMRIB, Oxford, United Kingdom). ${ }^{39}$ The statistical images

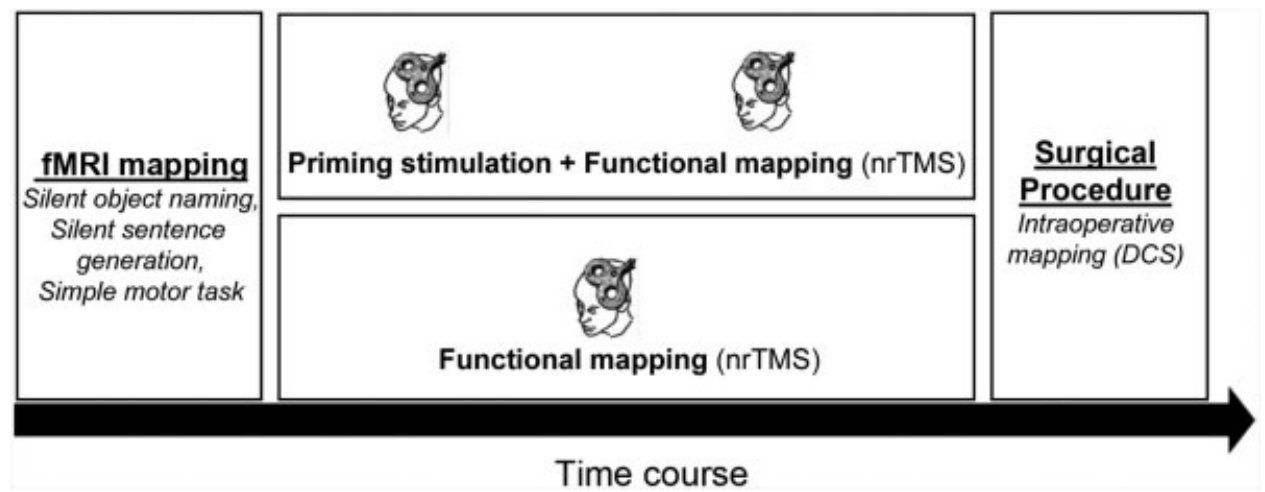

Fig. 1 Experimental time course. First, anatomical and functional magnetic resonance imaging (fMRI) data sets were obtained. Then the patient underwent a session of navigated repetitive transcranial magnetic stimulation (nrTMS) functional language mapping, either with or without priming stimulation in a randomized manner. The last part of the experiment involved the intraoperative language mapping using direct cortical stimulation (DCS) and the surgical removal of the tumor. 
were first thresholded by $z=3.1$ with a cluster significance threshold of $p=0.01$. After analyzing the fMRI paradigms separately, the resulting statistical images were integrated into a single combined statistical analysis to generate one functional image for the language network. For the combined analysis, 3D cope images from the separate first-level analysis were fed into one higher level analysis using a fixed effects model. The images were thresholded at $z=7$ with a corrected significance threshold of $p=0.01$.

\section{Preoperative nrTMS Language Mapping}

\section{Experimental Set-up}

The nrTMS language mapping experiments were performed using two devices (investigational use approved by the Food and Drug Administration), namely the MagPro X100 magnetic stimulator with MagOption (MagVenture $\mathrm{GmbH}$, Farum, Denmark) and Localite TMS Navigator (Localite $\mathrm{GmbH}$, St. Augustin, Germany) equipped with an infrared tracking device (Polaris Spectra, Northern Digital Inc., Waterloo, Ontario, Canada). A figure-of-eight MagVenture coil (MCF-B65) with 75-mm diameter on one winding was used to deliver single and repetitive biphasic TMS pulses.

\section{Coregistration Procedure}

The preoperative nrTMS language mapping began with the coregistration of the patient's head and the patient's 3D T1weighted MRI scan. The acquired 3D MRI image was uploaded to the Localite TMS Navigator System. For the tracking device to track the patient head positions during language mapping, three adhesive reflective sphere markers were attached to the patient's forehead. Subsequently, three anatomical landmarks (nasion and bilateral outer canthi) were marked in the 3D MRI image. Corresponding anatomical landmarks were marked on the patient's face with a digitizing pen that also contained three reflective sphere markers to enable the software to further correlate the spatial position of the device equipped with reflective markers to its position in relation to the patient's MRI. ${ }^{40}$

To improve the precision of the coregistration, 200 anatomical landmarks were added by tracing the patient's head with the digitizing pen. We kept the root mean squared error of the fitting procedure at $<2.5 \mathrm{~mm}$ for all patients to ensure the goodness of fit (patient's real head and structural MRI). The coregistration produced a $3 \mathrm{D}$ reconstruction of the patient's brain in which the peeling depth could be adjusted individually to visualize the brain cortical anatomy including the tumor. Afterward, the individual patient's functional data set was overlaid on the 3D reconstruction. Cortical areas with significant BOLD activations during tongue movements and language tasks ("fMRI hotspots"), as well as other areas near the tumor that were being considered important for resection, were identified and marked (at least $10 \mathrm{~mm}$ apart) as regions of interest (ROIs) (-Table $\mathbf{1}$ ).

\section{Picture-naming Task and Baseline Testing}

Before the TMS procedures, the participants were asked to perform a picture-naming task, a modified version of the
Boston Naming Test. ${ }^{41,42}$ They were asked to name blackand-white line drawings of common living (e.g., dog) and nonliving (e.g., table) objects as quickly and correctly as possible. Fifty-four pictures were displayed on a 15-inch notebook using a presentation program (Neurobehavioral Systems, Inc., Berkeley, CA, USA) for 3 seconds (picture display time [PDT]) at a rate controlled by the examiner to give the patient ample time to identify the object. The distance between the screen and the patient's head was individually determined by considering comfort as well as the subjective good view. When naming the object on the picture, the patient had to use the phrase "this is a" before the object name. Unfamiliar or misnamed objects with synonymous names were excluded from the task to ensure that any errors observed during the actual language mapping were elicited by the stimulation. We only used pictures that were named correctly for stimulation frequency determination and language mapping procedures.

\section{Stimulation Intensity and Frequency Determination}

After the coregistration and baseline picture-naming task performance, we determined the individual patient's active motor threshold (AMT) from the primary motor cortex representation of the right first dorsal interosseous (FDI) muscle using single-pulse TMS. Surface electrodes were placed at the right FDI muscle in a belly-tendon montage to monitor the motor-evoked potential (MEP) amplitudes during stimulation using the built-in electromyography (EMG) device in the stimulator.

Subsequently, we located the "hand knob" area in the left precentral gyrus using the patient's anatomical and fMRI data. All participants had a well-defined hand knob area in the left motor cortex that exhibited significant task-related activations. With the help of the neuronavigation system, the coil was placed tangentially to the scalp, always perpendicular to the hand knob with the handle pointing laterally and posteriorly, generating an anteroposterior current direction in the brain. Single-pulse TMS stimulation was applied at the hand knob area with an intensity set at $45 \%$ of the maximum stimulator output (MSO) and a frequency of $0.25 \mathrm{~Hz}$.

The stimulator output intensity was then adjusted (increased or decreased) and the coil position moved until the "motor hotspot" of the FDI muscle was localized. The motor hotspot is defined as the cortical location where the lowest stimulator output can elicit a maximal stable motor response with an amplitude of $1.0 \mathrm{mV}$ in the relaxed FDI muscle. The motor hotspot was marked on the patient's brain image. After localization of the motor hotspot, the patients were asked to contract the FDI muscle briefly and voluntarily ( $10 \%$ of the maximum voluntary contraction) while TMS was delivered. The stimulator output intensity was then decreased until the AMT or the minimum intensity that elicits an MEP response $\geq 100 \mu \mathrm{V}$ (peak to peak) in at least 5 of 10 consecutive trials was achieved. ${ }^{43}$ A stimulator intensity equivalent to $90 \%$ of the AMT was designated as the stimulation intensity for the language mapping.

We decided to use the AMT for language mapping for three reasons. (1) Stimulation intensity set at AMT is lower 
Table 1 Results of preoperative fMRI and nrTMS language mapping

\begin{tabular}{|c|c|c|c|c|}
\hline Patient & ROIs & $\begin{array}{l}\text { Inclusion criteria: } \\
\text { task-dependent (fMRI) } \\
\text { activation or } \\
\text { anatomical location }\end{array}$ & $\begin{array}{l}\text { Errors: Unprimed } \\
\text { nrTMS }\end{array}$ & $\begin{array}{l}\text { Errors: Primed } \\
\text { nrTMS }\end{array}$ \\
\hline \multirow[t]{10}{*}{ B1 } & 1-PCG (hand knob) & $\mathrm{Ft}$ & & \\
\hline & 2-PCG (hand knob) & $\mathrm{Ft}$ & & \\
\hline & 3-PCG (tongue) & SON, SSG & G & G \\
\hline & 4-PCG (tongue) & SON, SSG & G & G \\
\hline & 5-PCG (tongue) & SON, SSG & $A$ & G \\
\hline & 6-PMA & SON, SSG & G & G \\
\hline & 7-PMA & SON, SSG & G & G \\
\hline & 8-MFG & SON, SSG & A & G \\
\hline & 9-MFG & SON, SSG & $\mathrm{E}$ & G \\
\hline & 10-PCG (tongue) & SON, SSG & G & $\mathrm{D}$ \\
\hline \multirow[t]{13}{*}{ B2 } & 1-PCG (hand knob) & $\mathrm{Ft}$ & & \\
\hline & 2-PCG (hand knob) & $\mathrm{Ft}$ & & \\
\hline & 3-SMG & SON & G & G \\
\hline & 4-SMG & SON & G & G \\
\hline & 5-MFG & SON & G & G \\
\hline & 6-PMA & SON & G & G \\
\hline & 7-PMA & SON & B & G \\
\hline & 8-IFG & Anatomical location & G & G \\
\hline & 9-IFG & Anatomical location & G & G \\
\hline & 10-IFG & Anatomical location & G & G \\
\hline & 11-IFG & Anatomical location & G & G \\
\hline & 12-MFG & SON & G & G \\
\hline & 13-MFG & SON & G & G \\
\hline \multirow[t]{18}{*}{ B3 } & 1-PCG (hand knob) & $\mathrm{Ft}$ & & \\
\hline & 2-PCG (hand knob) & $\mathrm{Ft}$ & & \\
\hline & 3-PCG (hand knob) & $\mathrm{Ft}$ & & \\
\hline & 4-PCG (hand knob) & $\mathrm{Ft}$ & & \\
\hline & 5-PMA & $\mathrm{Ft}$ & G & G \\
\hline & 6-PMA & Anatomical location & G & G \\
\hline & 7-MFG & SSG & G & G \\
\hline & 8-PCG & SSG & G & G \\
\hline & 9-PMA & Anatomical location & G & G \\
\hline & 10-MFG & Anatomical location & $A$ & G \\
\hline & 11-MFG & Anatomical location & G & G \\
\hline & 12-PCG & Anatomical location & G & G \\
\hline & 13-PCG & Anatomical location & G & G \\
\hline & $14-A G$ & Anatomical location & G & $\mathrm{E}$ \\
\hline & 15-SMG & SSG & G & G \\
\hline & 16-PMA & -anatomical location & G & G \\
\hline & 17-PMA & -anatomical location & G & G \\
\hline & 18-PCG & -anatomical location & G & G \\
\hline
\end{tabular}


Table 1 (Continued)

\begin{tabular}{|c|c|c|c|c|}
\hline Patient & ROIs & $\begin{array}{l}\text { Inclusion criteria: } \\
\text { task-dependent (fMRI) } \\
\text { activation or } \\
\text { anatomical location }\end{array}$ & $\begin{array}{l}\text { Errors: Unprimed } \\
\text { nrTMS }\end{array}$ & $\begin{array}{l}\text { Errors: Primed } \\
\text { nrTMS }\end{array}$ \\
\hline & 19-MFG & -anatomical location & G & G \\
\hline & 20-MFG & -anatomical location & $G$ & $G$ \\
\hline & 21-MFG & SSG & $G$ & $G$ \\
\hline & 22-SMG & SSG & $G$ & $G$ \\
\hline & 23-IFG & -anatomical location & A & G \\
\hline \multirow[t]{14}{*}{ B4 } & 1-PCG (hand knob) & $\mathrm{Ft}$ & & \\
\hline & 2-PCG (hand knob) & $\mathrm{Ft}$ & & \\
\hline & 3-PCG (hand knob) & $\mathrm{Ft}$ & & \\
\hline & 4-SMG & SON, SSG & B & G \\
\hline & 5-SMG & -anatomical location & $\mathrm{B}$ & $G$ \\
\hline & 6-AG & SON, SSG & $G$ & $G$ \\
\hline & 7-IFG & SON, SSG & $G$ & $G$ \\
\hline & 8-IFG & SON, SSG & B & G \\
\hline & 9-IFG & SON, SSG & B & G \\
\hline & 10-IFG & SON, SSG & A & $G$ \\
\hline & 11-POCG & -anatomical location & $B$ & $G$ \\
\hline & 12-PCG (tongue) & TM & $E$ & B \\
\hline & 13-PCG (tongue) & TM & E & B \\
\hline & 14-PMA & SON,SSG & $G$ & $G$ \\
\hline \multirow[t]{22}{*}{ B5 } & 1-PCG (hand knob) & $\mathrm{Ft}$ & & \\
\hline & 2-PCG (hand knob) & $\mathrm{Ft}$ & & \\
\hline & 3-PCG (hand knob) & $\mathrm{Ft}$ & & \\
\hline & 4-PCG (hand knob) & $\mathrm{Ft}$ & & \\
\hline & 5-PCG (hand knob) & $\mathrm{Ft}$ & & \\
\hline & 6-IFG & SON, SSG & G & G \\
\hline & 7-PMA & SON, SSG & G & G \\
\hline & 8-PMA & SON, SSG & $G$ & $G$ \\
\hline & 9-PMA & SON, SSG & G & B \\
\hline & 10-PMA & SON, SSG & $G$ & $G$ \\
\hline & 11-IFG & SON, SSG & G & G \\
\hline & 12-IFG & SON, SSG & G & G \\
\hline & 13-IFG & SON, SSG & $G$ & $G$ \\
\hline & 14-IFG & SON, SSG & A & $G$ \\
\hline & 15-IFG & SON, SSG & G & G \\
\hline & 16-SMG & SON, SSG & G & G \\
\hline & 17-MTC & SON, SSG & $G$ & $G$ \\
\hline & 18-PMA & SON, SSG & $G$ & $G$ \\
\hline & 19-OL & -anatomical location & G & G \\
\hline & 20-POCG & $\mathrm{TM}$ & $G$ & $G$ \\
\hline & 21-PCG & $\mathrm{TM}$ & $G$ & $G$ \\
\hline & 22-IFG & -anatomical location & G & G \\
\hline
\end{tabular}


Table 1 (Continued)

\begin{tabular}{|c|c|c|c|c|}
\hline Patient & ROIs & $\begin{array}{l}\text { Inclusion criteria: } \\
\text { task-dependent (fMRI) } \\
\text { activation or } \\
\text { anatomical location }\end{array}$ & $\begin{array}{l}\text { Errors: Unprimed } \\
\text { nrTMS }\end{array}$ & $\begin{array}{l}\text { Errors: Primed } \\
\text { nrTMS }\end{array}$ \\
\hline & 23-IFG & -anatomical location & G & G \\
\hline & 24-IFG & -anatomical location & G & G \\
\hline & 25-SMG & -anatomical location & G & G \\
\hline \multirow[t]{18}{*}{ B6 } & 1-PCG (hand knob) & $\mathrm{Ft}$ & & \\
\hline & 2-PCG (hand knob) & $\mathrm{Ft}$ & & \\
\hline & 3-PCG (hand knob) & $\mathrm{Ft}$ & & \\
\hline & 4-PCG (hand knob) & $\mathrm{Ft}$ & & \\
\hline & 5-PMA & SON, SSG & G & G \\
\hline & 6-PMA & SON, SSG & G & G \\
\hline & 7-PMA & SON, SSG & $G$ & $G$ \\
\hline & 8-PMA & SON, SSG & G & G \\
\hline & 9-PMA & SON, SSG & $A$ & $G$ \\
\hline & 10-PMA & SON, SSG & $G$ & $G$ \\
\hline & 11-PMA & -anatomical location & $A$ & G \\
\hline & 12-PCG & SON, SSG & G & $G$ \\
\hline & 13-PCG & -anatomical location & $G$ & $G$ \\
\hline & 14-POCG & TM & G & G \\
\hline & 15-POCG & -anatomical location & $A$ & $G$ \\
\hline & 16-PMA & SON, SSG & G & $G$ \\
\hline & 17-PMA & SON, SSG & $G$ & G \\
\hline & 18-PMA & SON, SSG & G & G \\
\hline \multirow[t]{20}{*}{ B7 } & 1-PCG (hand knob) & $\mathrm{Ft}$ & & \\
\hline & 2-PCG (hand knob) & $\mathrm{Ft}$ & & \\
\hline & 3-PCG (hand knob) & $\mathrm{Ft}$ & & \\
\hline & 4-PMA & SSG & G & $G$ \\
\hline & 5-PMA & SON, SSG & $E$ & $G$ \\
\hline & 6-PMA & SON, SSG & G & G \\
\hline & 7-PMA & SON, SSG & $G$ & $G$ \\
\hline & 8-IFG & SON, SSG & $G$ & $G$ \\
\hline & 9-POCG & TM & $G$ & $G$ \\
\hline & 10-POCG & TM & $G$ & $G$ \\
\hline & 11-PCG & TM & $G$ & $\mathrm{~B}$ \\
\hline & 12-PCG & TM & G & G \\
\hline & 13-PL & TM & G & G \\
\hline & 14-IFG & SSG & G & G \\
\hline & 15-MFG & SSG & $G$ & $G$ \\
\hline & 16-PL & SSG & G & G \\
\hline & 17-PL & SSG & B & $G$ \\
\hline & $18-\mathrm{PL}$ & SSG & $E$ & $G$ \\
\hline & 19-PL & SSG & G & G \\
\hline & $20-\mathrm{PL}$ & SSG & $G$ & $G$ \\
\hline
\end{tabular}


Table 1 (Continued)

\begin{tabular}{|c|c|c|c|c|}
\hline Patient & ROIs & $\begin{array}{l}\text { Inclusion criteria: } \\
\text { task-dependent (fMRI) } \\
\text { activation or } \\
\text { anatomical location }\end{array}$ & $\begin{array}{l}\text { Errors: Unprimed } \\
\text { nrTMS }\end{array}$ & $\begin{array}{l}\text { Errors: Primed } \\
\text { nrTMS }\end{array}$ \\
\hline \multirow[t]{30}{*}{ B8 } & 1-PCG (hand knob) & $\mathrm{Ft}$ & & \\
\hline & 2-PCG (hand knob) & $\mathrm{Ft}$ & & \\
\hline & 3-PCG (hand knob) & $\mathrm{Ft}$ & & \\
\hline & 4-PMA & SSG & G & G \\
\hline & 5-PMA & SSG & $G$ & $G$ \\
\hline & 6-PMA & SON, SSG & G & G \\
\hline & 7-PMA & SON, SSG & $G$ & $G$ \\
\hline & 8-PMA & SON, SSG & G & G \\
\hline & 9-MFG & SON, SSG & G & G \\
\hline & 10-MFG & SON, SSG & $G$ & $G$ \\
\hline & 11-IFG & SON, SSG & $G$ & $\mathrm{E}$ \\
\hline & 12-IFG & SON, SSG & $G$ & $G$ \\
\hline & 13-MFG & SON, SSG & G & $\mathrm{E}$ \\
\hline & 14-IFG & SON, SSG & $E$ & G \\
\hline & 15-IFG & SON, SSG & $\mathrm{E}$ & G \\
\hline & 16-PL & SON, SSG & $G$ & $G$ \\
\hline & 17-PCG & TM & $E$ & G \\
\hline & 18-PCG & TM & G & G \\
\hline & 19-IFG & SSG & G & G \\
\hline & 20-PMA & Anatomical location & $G$ & $G$ \\
\hline & 21-PMA & Anatomical location & G & G \\
\hline & 22-PMA & Anatomical location & G & G \\
\hline & 23-PMA & Anatomical location & G & G \\
\hline & 24-PMA & Anatomical location & G & G \\
\hline & 25-PMA & Anatomical location & G & G \\
\hline & 26-PMA & Anatomical location & G & G \\
\hline & 27-PMA & Anatomical location & G & G \\
\hline & 28-POCG & Anatomical location & G & G \\
\hline & 29-PMA & Anatomical location & G & G \\
\hline & 30-PMA & Anatomical location & G & G \\
\hline \multirow[t]{11}{*}{ B9 } & 1-PCG (hand knob) & $\mathrm{Ft}$ & & \\
\hline & 2-PCG (hand knob) & $\mathrm{Ft}$ & & \\
\hline & 3-PCG (hand knob) & $\mathrm{Ft}$ & & \\
\hline & 4-PCG (hand knob) & $\mathrm{Ft}$ & & \\
\hline & 5-PMA & SSG & G & G \\
\hline & 6-IFG & Anatomical location & $G$ & $G$ \\
\hline & 7-IFG & SSG & $G$ & $G$ \\
\hline & 8-IFG & SSG & G & G \\
\hline & 9-STG & SSG & G & G \\
\hline & 10-PCG & $\mathrm{TM}$ & $G$ & $G$ \\
\hline & 11-PCG & TM & G & G \\
\hline
\end{tabular}


Table 1 (Continued)

\begin{tabular}{|c|c|c|c|c|}
\hline Patient & ROIs & $\begin{array}{l}\text { Inclusion criteria: } \\
\text { task-dependent (fMRI) } \\
\text { activation or } \\
\text { anatomical location }\end{array}$ & $\begin{array}{l}\text { Errors: Unprimed } \\
\text { nrTMS }\end{array}$ & $\begin{array}{l}\text { Errors: Primed } \\
\text { nrTMS }\end{array}$ \\
\hline & 12-PMA & SON & $G$ & G \\
\hline & 13-PMA & SON & $G$ & $G$ \\
\hline & 14-PMA & Anatomical location & G & G \\
\hline & 15-POCG & Anatomical location & G & G \\
\hline & 16-POCG & Anatomical location & $G$ & $G$ \\
\hline & 17-POCG & Anatomical location & G & $G$ \\
\hline & 18-POCG & Anatomical location & $G$ & $G$ \\
\hline & 19-STG & Anatomical location & G & G \\
\hline & $20-A G$ & Anatomical location & G & G \\
\hline & 21-ATG & Anatomical location & $G$ & $G$ \\
\hline & 22-STG & Anatomical location & $E$ & $G$ \\
\hline & 23-PMA & Anatomical location & $E$ & $G$ \\
\hline \multirow[t]{22}{*}{ B10 } & 1-PCG (hand knob) & $\mathrm{Ft}$ & & \\
\hline & 2-PCG (hand knob) & $\mathrm{Ft}$ & & \\
\hline & 3-PCG (hand knob) & $\mathrm{Ft}$ & & \\
\hline & 4-PCG (hand knob) & $\mathrm{Ft}$ & & \\
\hline & 5-PCG (hand knob) & $\mathrm{Ft}$ & & \\
\hline & 6-PMA & SON, SSG & $\mathrm{B}$ & $G$ \\
\hline & 7-PMA & SON, SSG & G & G \\
\hline & 8-IFG & SON, SSG & G & G \\
\hline & 9-PMA & SON, SSG & G & G \\
\hline & 10-PL & SON, SSG & G & G \\
\hline & $11-\mathrm{PL}$ & SON, SSG & $G$ & $G$ \\
\hline & 12-MFG & SON, SSG & G & G \\
\hline & 13-PMA & SON, SSG & $E$ & G \\
\hline & 14-PL & SON, SSG & $G$ & $G$ \\
\hline & 15-PL & SON, SSG & G & G \\
\hline & 16-PL & -Anatomical location & $G$ & $G$ \\
\hline & 17-PL & -Anatomical location & G & $G$ \\
\hline & 18-PCG & -Anatomical location & G & $G$ \\
\hline & 19-PL & -Anatomical location & $G$ & $G$ \\
\hline & 20-PMA & SON, SSG & $G$ & $G$ \\
\hline & 21-IFG & SON, SSG & G & G \\
\hline & 22-PCG & SON, SSG & G & G \\
\hline \multirow[t]{7}{*}{ B11 } & 1-PCG (hand knob) & $\mathrm{Ft}$ & & \\
\hline & 2-PCG (hand knob) & $\mathrm{Ft}$ & & \\
\hline & 3-PCG (hand knob) & $\mathrm{Ft}$ & & \\
\hline & 4-PCG (hand knob) & $\mathrm{Ft}$ & & \\
\hline & 5-PCG (hand knob) & $\mathrm{Ft}$ & & \\
\hline & 6-PCG & TM & $\mathrm{E}$ & G \\
\hline & 7-PCG & TM & $G$ & $G$ \\
\hline
\end{tabular}


Table 1 (Continued)

\begin{tabular}{|c|c|c|c|c|}
\hline Patient & ROls & $\begin{array}{l}\text { Inclusion criteria: } \\
\text { task-dependent (fMRI) } \\
\text { activation or } \\
\text { anatomical location }\end{array}$ & $\begin{array}{l}\text { Errors: Unprimed } \\
\text { nrTMS }\end{array}$ & $\begin{array}{l}\text { Errors: Primed } \\
\text { nrTMS }\end{array}$ \\
\hline & 8-PMA & SSG & G & G \\
\hline & 9-PMA & SSG & $G$ & G \\
\hline & 10-PMA & SSG & $G$ & G \\
\hline & 11-IFG & SON & G & G \\
\hline & 12-IFG & SON & $\bar{G}$ & G \\
\hline & 13-PMA & SON & $G$ & $G$ \\
\hline & 14-SMG & SON & G & G \\
\hline & 15-STG & SON & G & G \\
\hline & 16-PMA & Anatomical location & G & G \\
\hline & 17-PMA & Anatomical location & G & G \\
\hline & 18-PMA & SON,SSG & G & G \\
\hline \multirow[t]{21}{*}{ B12 } & 1-PCG (hand knob) & $\mathrm{Ft}$ & & \\
\hline & 2-PCG (hand knob) & $\mathrm{Ft}$ & & \\
\hline & 3-PCG (hand knob) & $\mathrm{Ft}$ & & \\
\hline & 4-PCG (hand knob) & $\mathrm{Ft}$ & & \\
\hline & 5-PCG (hand knob) & $\mathrm{Ft}$ & & \\
\hline & 6-PCG (hand knob) & $\mathrm{Ft}$ & & \\
\hline & 7-SMG & SON, SSG & G & G \\
\hline & 8-SMG & Anatomical location & G & G \\
\hline & 9-IFG & SON, SSG & A & G \\
\hline & 10-MFG & SON, SSG & G & G \\
\hline & 11-MFG & SON, SSG & $G$ & $G$ \\
\hline & 12-MFG & SON, SSG & G & G \\
\hline & 13-MFG & Anatomical location & A & G \\
\hline & 14-MFG & Anatomical location & G & G \\
\hline & 15-IFG & Anatomical location & G & G \\
\hline & 16-IFG & SON, SSG & A & G \\
\hline & $17-A G$ & SON, SSG & G & G \\
\hline & 18-PCG & SON, SSG & G & G \\
\hline & 19-PCG & SON, SSG & G & G \\
\hline & 20-PMA & Anatomical location & $E$ & G \\
\hline & 21-IFG & Anatomical location & G & G \\
\hline
\end{tabular}

This table shows the brain areas significantly recruited during fMRI language mapping and language errors elicited during unprimed and primed nrTMS mapping in individual patient.

Abbreviations: A, no response; AG, angular gyrus; B, hesitation; BOLD, blood oxygen dependent; C, circumlocution; D, semantic paraphasia; E, phonological paraphasia; F, neologism; fMRI, functional magnetic resonance imaging; Ft, finger tapping task; G, normal response; IFG, inferior frontal gyrus; ITG, inferior temporal gyrus; MFG, middle frontal gyrus; MT, tongue movement; MTG, middle temporal gyrus; nrTMS, navigated repetitive transcranial magnetic stimulation; PCG, precentral gyrus; PL, parietal lobe; PMA, premotor area; POCG, postcentral gyrus; ROI, region of interest; SFG, superior frontal gyrus; SMG, supramarginal gyrus; SON, silent object-name task; SSG, silent sentence generation task; STG, superior temporal gyrus. 
than the commonly used resting motor threshold (RMT) and therefore causes less discomfort, particularly during the stimulation near or at the inferior frontal gyrus. (2) Speech production increases motor excitability not only in the face area but also in the hand area. ${ }^{44}$ AMT, which closely indexes the excitability of active motor cortex neurons, therefore resembles the excitability of language-eloquent cortical neurons when they are engaged in a task. This is a more representative setting than RMT, which is recorded from a resting hand muscle. (3) The use of the AMT is also consistent with the original inhibitory paradigm of cTBS. ${ }^{16}$

After identifying the stimulation intensity, we determined the stimulation frequency from the primary motor cortex representation of the tongue muscle. From the motor hotspot of the FDI muscle, the magnetic coil was slowly moved laterally in 5-mm steps while the patients were performing number counting from 1 to 10 . During this procedure, cTBS was administered at different frequencies (4 Hz, 5 pulses, 90\% AMT; $5 \mathrm{~Hz}, 5$ pulses, 90\% AMT; $7 \mathrm{~Hz}, 5$ pulses, 90\% AMT, and $7 \mathrm{~Hz}, 7$ pulses, 90\% AMT). ${ }^{10,45}$ If no stammering or tactile sensations in the tongue (e.g., heaviness) was observed or reported by the patient, the procedure was repeated using the picture-naming task. For the picturenaming task, the picture to trigger time (PTT) was set to $0 \mathrm{~ms}$, resulting in a direct start of the stimulation at the presentation of each picture, and the PDT was set to 3 seconds.

By using codes, the neuropsychologist categorized the patient's response into the following categories: A, if the stimulation led to a complete lack (no response) of the naming response; $\mathrm{B}$, if the stimulation led to a 1-second delay ("hesitation") in the naming response; C, "circumlocution" when the subject talked about or around the target word instead of naming it (e.g., the target word "chair" is replaced with " sit down"); D, "semantic paraphasia” when the patient substituted a semantically related or associated word for the target word (e.g., target word "cow" is replaced by the word "horse"); E, "phonological paraphasia" when the patient made phonemic modifications of the target word (e.g., the target word "pants" is replaced with "plants"); F, "neologism" when the patient made form-based errors that are nonexistent words (e.g., target word "horse" is replaced with the word "herp"); and G, normal response if the subject was able to name the object correctly. ${ }^{1,46}$ The frequency that elicited multiple language errors was identified and designated as the stimulation frequency for language mapping. If no errors were elicited, the stimulation intensity was increased by $10 \%$, and the procedure was repeated. Alternatively, if major discomfort was observed, the intensity was lowered in $10 \%$ steps until a tolerable level was reached. All stimulation parameters conformed to the safety guidelines for rTMS. ${ }^{38,47,48}$

\section{The nrTMS Language Mapping Procedure}

The patients underwent two nrTMS sessions: primed and unprimed. In the unprimed session, the patient immediately underwent language mapping after baseline testing. In the primed session, an identical stimulation paradigm (without a language task) was applied to all ROIs (fMRI hotspots and anatomically selected target areas) 15 minutes before the language mapping. The number of ROIs varied between patients and depended on the location and size of the tumor (-Tables 1 and 2). During the stimulation, the magnetic coil was held perpendicular to the targeted gyri to achieve maximum field induction. ${ }^{25}$ In both sessions, PTT and PDT were similar to those used during the stimulation frequency determination ( 0 seconds and 3 seconds, respectively) allowing one ROI to be stimulated three times before moving to the next ROI.

Three pictures were presented for each stimulated site. During the mapping, a stimulation site was considered "language positive" when an error occurred in at least two of three pictures during stimulation. Subsequently, an oral assessment was performed before moving to the next ROI. Patients were asked whether they were aware of the error that they committed and asked if factors other than the stimulation could have affected their response. Most

Table 2 Demographic data and clinical characteristics of the 12 patients enrolled in the study

\begin{tabular}{|c|c|c|c|c|}
\hline Patient & Age, y & Sex & Tumor type & Tumor location \\
\hline B1 & 52 & $\mathrm{~F}$ & Astrocytoma, IDH-mutated, WHO ${ }^{\circ} \mathrm{I}$ & Left frontal lobe \\
\hline B2 & 69 & $\mathrm{~F}$ & Oligodendroglioma, IDH-mutated, 1p19q codeleted, WHO ${ }^{\circ} \mathrm{I}$ & Left fronto-insular \\
\hline B3 & 52 & M & Anaplastic astrocytoma, IDH-mutated, WHO ${ }^{\circ} \mathrm{III}$ & Left frontal lobe \\
\hline B4 & 32 & M & Astrocytoma, IDH-wildtype, WHO ${ }^{\circ} \mathrm{II}-\mathrm{III}$ & Left temporal lobe \\
\hline B5 & 29 & M & Astrocytoma, IDH-mutated, WHO ${ }^{\circ} \mathrm{I}$ & Left fronto-temporo-insular \\
\hline B6 & 32 & M & Oligodendroglioma, IDH-mutated, 1p19q co-deleted, WHO II & Left fronto-temporo-insular \\
\hline B7 & 58 & $\mathrm{M}$ & Oligodendroglioma, IDH-mutated, 1p19q co-deleted, WHO `II & Left fronto-insular \\
\hline B8 & 40 & M & Oligodendroglioma, IDH-mutated, 1p19q co-deleted, WHO `II & Left frontal lobe \\
\hline B9 & 58 & $\mathrm{~F}$ & Glioblastoma, IDH-wild type, WHO ${ }^{\circ} \mathrm{IV}$ & Left fronto-insular \\
\hline B10 & 20 & $\mathrm{~F}$ & Pilocytic astrocytoma, $\mathrm{WHO}{ }^{\circ}$ & Left parietal lobe \\
\hline B11 & 40 & $\mathrm{~F}$ & Astrocytoma, IDH-mutated, WHO ${ }^{\circ} \mathrm{I}$ & Left frontal lobe \\
\hline B12 & 31 & $\mathrm{~F}$ & Oligodendroglioma, IDH-mutated, 1p19q co-deleted, WHO ${ }^{\circ}$ & Left frontal lobe \\
\hline
\end{tabular}

Abbreviations: IDH, isocitrate dehydrogenase; WHO, World Health Organization. 
importantly, the patients were assured that errors such as speech arrest would be temporary. On the other hand, a stimulation site was considered "language negative" when errors occurred in only one or none of the pictures during stimulation. Cortical sites where only one error occurred were marked and restimulated to confirm that the response was reproducible. The occurrence of only one or no error confirmed that the site was language negative. On the other hand, eliciting more errors during restimulation could change the category of the site into language positive. The patient's response was video recorded so the neuropsychologist could reanalyze the errors offline if needed.

\section{Intraoperative DCS Language Mapping during Awake Surgery}

A modified asleep-awake-asleep set-up was used during the operation. In the first asleep phase, the patients were under general anesthesia (propofol $2-4 \mu \mathrm{g} / \mathrm{kg} / \mathrm{min}$ plus remifentanil $0.02-0.05 \mathrm{mg} / \mathrm{kg}$ or $50-100 \mathrm{mg}$ fentanyl bolus) using a target-controlled infusion system. The depth of anesthesia was measured using the bispectral index. Set-up, craniotomy, and determination of stimulation intensity were all performed during the first asleep phase.

Functional language mapping and tumor resection were then performed during the awake phase, lasting as long as necessary. Intraoperative language mapping began with the identification of language-positive areas in the vicinity of the tumor. DCS was performed using a bipolar stimulation electrode (ISIS IOM system, Inomed Medizintechnik, Emmendingen, Germany) under free-running EMG (musculus [M.] orbicularis oris, M. extensor digitorum, M. abductor pollicis brevis, M. tibialis anterior, and M. abductor hallucis longus) and EEG provided by a cortical strip electrode. The bipolar stimulation electrode had a $5-\mathrm{mm}$ interelectrode distance, and a monophasic current was delivered (square wave with a single-pulse phase duration of $1.0 \mathrm{~ms}$ ) in a 4-s train at $30 \mathrm{~Hz}$. Stimulation intensity was started at $1 \mathrm{~mA}$ and gradually increased up to a maximum of $6 \mathrm{~mA}$ until afterdischarge potentials were detected at the strip electrode over the motor cortex. ${ }^{49}$ The same set of pictures for preoperative nrTMS mapping was also used for the intraoperative mapping. The PDT was variable and depended on the patient's response time and intraoperative modalities.

The picture presentation and stimulation synchronization were achieved through the use of an audio signal (a short click) produced from the tablet PC on the appearance of every picture. The audio signal allows the surgeon to synchronize the stimulation with the picture-naming task. Typically, not more than $190 \mathrm{~ms}$ were anticipated for the delay between the audio signal and the surgeon's response. Each cortical target area was stimulated three times following the two out of three rule. ${ }^{50}$ All patients were asked to use the phrase "this is a ..." before the object name during the stimulation. Similar to TMS, the language-positive site was defined as an area that induced language errors or speech arrest when stimulated. ${ }^{51}$

To avoid any bias, the TMS mapping results were not available to the surgeon during DCS. Once a target spot was identified to be language positive, it was marked with sterile paper numbers. Then its location was marked and saved into the navigation system using a navigational pointer. Tumor resection and subcortical mapping were done in the usual fashion. The mapped cortical surface was photographed before and after tumor resection. The final steps of the surgery were completed during the second asleep phase. Dexmedetomidine $(0.2-0.5 \mathrm{~g} / \mathrm{kg} / \mathrm{h})$ was used as an adjunct drug to propofol for sedation during this phase.

\section{Data Analysis}

\section{TMS Data Analysis}

Statistical analysis was performed using SPSS software v.22 (IBM Corp., Armonk, New York, United States). Errors in TMSpositive sites were grouped into two categories: "major errors," such as speech arrests and hesitations, and "minor errors," such as circumlocutions, semantic paraphasias, phonological paraphasias, or neologisms. The error rates (ERs) were calculated separately for each error category within a session. We decided to calculate the ER because the number of target sites varied between patients. The number of nrTMS-positive language sites was subtracted from the total number of target sites. Then the difference was divided by the number of nrTMS-positive language sites and expressed in a percentage.

To explore our primary a priori objective of comparing the primed and unprimed nrTMS session, a repeated measure analysis of variance (ANOVA) was performed using the ER from each session as the dependent variable. Error type (major versus minor) and session type (primed versus unprimed) served as the within-subject factors. Sphericity was tested, and the Greenhouse-Geisser correction applied when necessary. Significant findings from the ANOVA were explored using post hoc comparisons (paired $t$ test, twotailed, Bonferroni adjusted for multiple comparisons). A $p$ value $<0.05$ was considered significant for all statistical analyses. All values were expressed as mean \pm SEM.

\section{Intraoperative DCS Assessment}

During the intraoperative assessment, positive findings for the motor and sensory mappings included movement or dysesthesias, respectively, during stimulation. ${ }^{52}$ For language mapping, the surgical team marked an area as language positive when a language error or deficit was elicited by DCS stimulation. The comparison of nrTMS and DCS results was performed visually by reimporting data generated intraoperatively into the TMS navigation system. Both results were compared according to their spatial distribution and were categorized based on the approach using the anatomical cortical parcellation system. Responses were categorized as true-positive responses when both DCS and nrTMS elicited language errors in target areas within $5 \mathrm{~mm}$ from each other and true-negative responses when both DCS and nrTMS did not elicit any speech disturbance. Falsepositive responses occurred when nrTMS impaired speech without any positive DCS confirmation; and false-negative responses when DCS elicited a positive but nrTMS elicited a negative response. ${ }^{1}$ 


\section{Results}

\section{Demographics and Tumor Characteristics}

The study was completed within a 16-month period from November 2016 to February 2018. All patients were native German speakers and at least 18 years of age at the time of the study. Ten patients were newly diagnosed; two patients had a recurrence. None of them were on antiepileptic drugs during the experiments. The postoperative histologic diagnosis was grade II oligodendroglioma in four cases, grade II astrocytoma in three cases, diffuse low-grade glioma in four cases, and pilocytic astrocytoma in one case (-Table 2 ).

\section{Preoperative fMRI, Baseline Picture-naming Testing, and nrTMS Tolerability}

The preoperative fMRI language mapping procedure identified 113 sites specific to language tasks (-Table 1). Overall, $66.37 \%$ of the sites were significantly recruited by both tasks (silent object naming and silent sentence generation), $20.35 \%$ by the silent sentence generation task, and $13.27 \%$ by the silent object-naming task alone. Because of their proximity to the tumor location, 16 sites significantly recruited by tongue movements and 61 sites anatomically classified to be relevant for later resections were added as ROIs. In total, we designated 190 ROIs or target sites (excluding the 47 areas recruited by finger tapping) for the nrTMS language mapping (-Table 1). During the baseline picturenaming task, all patients were able to identify the 54 presented pictures correctly. Therefore, all pictures were included in the picture-naming task used for stimulation frequency determination and nrTMS mapping.

The nrTMS stimulation was performed using the individually determined $90 \%$ of the AMT and a pulse train of 5 pulses at $5 \mathrm{~Hz}$ (-Table 3). These stimulation parameters, although not effective in disrupting number counting, were the most effective in eliciting errors in the picture-naming task during the motor mapping of the tongue muscle. The mean stimulation intensity during the primed and unprimed nrTMS session was $39 \%$ and $41 \%$ (percentage of the MSO), respectively. Although the stimulation intensity was determined individually and set to the most comfortable value, seven patients reported discomfort due to strong facial muscle contractions, especially during frontal lobe stimulation. Therefore, some areas of the frontal lobe (e.g., the interior frontal gyrus were excluded as targets and not further tested on these patients. Overall, the patients tolerated the nrTMS stimulation well. No other adverse effects such as seizures, headaches, dizziness, nausea, and vomiting were observed.

\section{Preoperative nTMS Language Mapping}

The number of language errors elicited during nrTMS language mapping was low ( - Table 1 ). In the unprimed session, errors were elicited in $16.81 \%$ of the language task ROIs ( 19 of 113), $25 \%$ in ROIs recruited by tongue movements (4 of 16), and $16.39 \%$ in ROIs that were anatomically selected (10 of 61 ). Overall, the stimulation elicited errors from $17.37 \%$ of the total ROIs (33 of 190). Concerning the error type, the participants committed more major errors (60.61\%) than minor errors (39.39\%) during the unprimed session. Similarly, the total number of errors during the primed session was low with $4.21 \%$ of all ROIs ( 8 of 190). Errors were elicited in only $3.54 \%$ of the language task ROIs (4 of 113 ). However, $18.75 \%$ ( 3 of 16 ) and $1.64 \%$ ( 1 of 61 ) errors were elicited in ROIs recruited by tongue movements and ROIs that were anatomically selected, respectively.

In contrast to the unprimed session, more minor errors (62.5\%) than major errors (37.5\%) occurred during this session. The statistical analysis of the calculated ER revealed significant

Table 3 Stimulation parameters of nrTMS

\begin{tabular}{|l|l|l|l|}
\hline Patient & $\begin{array}{l}\text { Mapping intensity, } \\
\text { 90\% of AMT: } \\
\text { Unprimed session }\end{array}$ & $\begin{array}{l}\text { Mapping intensity, } \\
\text { 90\% of AMT: } \\
\text { Primed session }\end{array}$ & $\begin{array}{l}\text { Mapping } \\
\text { frequency, Hz }\end{array}$ \\
\hline B1 & 30.6 & 32.4 & $5 \mathrm{~Hz} / 5 \mathrm{pulses}$ \\
\hline B2 & 56.7 & 49.5 & $5 \mathrm{~Hz} / 5 \mathrm{pulses}$ \\
\hline B3 & 36 & 29.7 & $5 \mathrm{~Hz} / 5 \mathrm{pulses}$ \\
\hline B4 & 46.8 & 45 & $5 \mathrm{~Hz} / 5 \mathrm{pulses}$ \\
\hline B5 & 42.3 & 37.8 & $5 \mathrm{~Hz} / 5 \mathrm{pulses}$ \\
\hline B6 & 43.2 & 37.8 & $5 \mathrm{~Hz} / 5 \mathrm{pulses}$ \\
\hline B7 & 37.8 & 39.6 & $5 \mathrm{~Hz} / 5 \mathrm{pulses}$ \\
\hline B8 & 52.2 & 40.5 & $5 \mathrm{~Hz} / 5 \mathrm{pulses}$ \\
\hline B9 & 40.5 & 37.8 & $5 \mathrm{~Hz} / 5 \mathrm{pulses}$ \\
\hline B10 & 45 & 43.2 & $5 \mathrm{~Hz} / 5 \mathrm{pulses}$ \\
\hline B11 & 37.8 & 41.4 & $5 \mathrm{~Hz} / 5 \mathrm{pulses}$ \\
\hline B12 & 33.3 & 33.3 & $5 \mathrm{~Hz} / 5 \mathrm{pulses}$ \\
\hline
\end{tabular}

Abbreviations: AMT, active motor threshold; nrTMS, navigated repetitive transcranial magnetic stimulation.

Note: This table shows the stimulation parameters for the individual patient including the mapping intensity (90\% of the AMT) expressed as a percentage of the maximum stimulator output, mapping frequency $(\mathrm{Hz})$, and the number of pulses in a single stimulation train. 


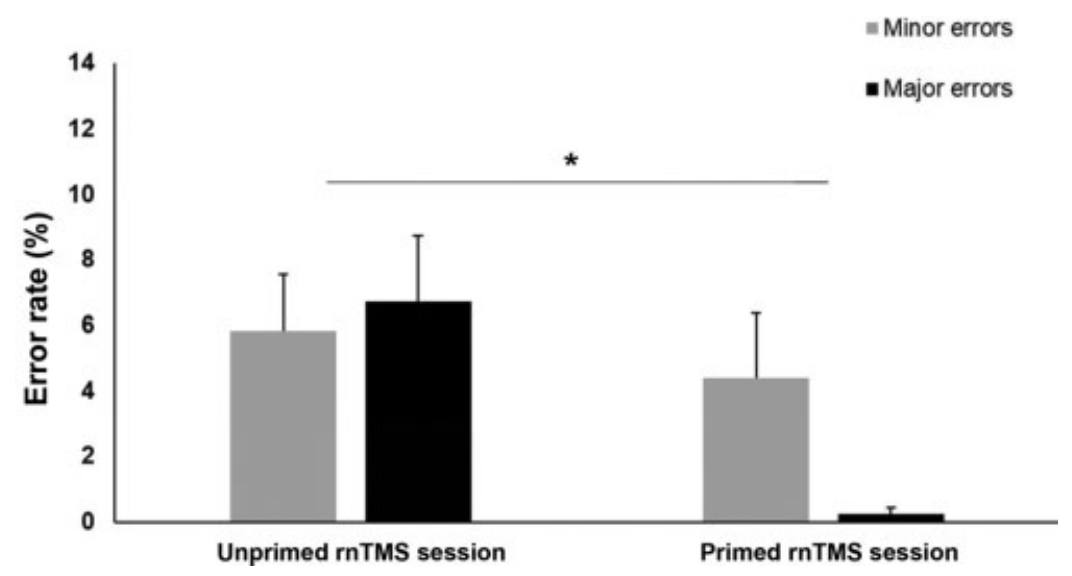

Fig. 2 The impact of primed and unprimed navigated repetitive transcranial magnetic stimulation (nrTMS) on the error rate (ER) during preoperative language mapping. The $x$-axis displays the error types in both unprimed and primed nrTMS sessions. The $y$-axis displays the ER in percentages. The graph shows the significant differences in the total ER in both sessions. Asterisk indicates significant differences between unprimed and primed total ER (Student $t$ test, paired, two tailed, $p<0.05$, Bonferroni corrected).

differences between the two nrTMS sessions (significant main effect of session: $\left.F(1,11)=6.03 ; p=0.032 ; \eta^{2}=0.354\right)$. The mean ER $( \pm \mathrm{SD})$ during the unprimed session $(6.27 \pm 3.88 \%)$ was significantly higher compared with the ER during the primed session $(2.31 \pm 3.36 \%)(-$ Fig. 2$)$. This result directly indicated there were more language-positive sites identified during the unprimed session. Furthermore, the overall main effect of error type on the ER was not significant $\left(F(1,11)=1.05 ; p=0.328 ; \eta^{2}=0.087\right)$. Although there were more major errors in the unprimed session and more minor errors in the primed session, the differences were not statistically significant (nonsignificant interaction of session and error type: $F(1,11)=1.53$; $\left.p=0.243 ; \eta^{2}=0.122\right)$.

\section{Comparison of nrTMS and DCS Language Maps}

In the present study, we only compared unprimed nrTMS and DCS because during both unprimed nrTMS and DCS, the cortex was stimulated only once. In primed nrTMS, cortical areas received two sets of stimulation (priming and real mapping stimulation). Naming errors were elicited by unprimed nrTMS as well as by DCS. In total, 190 cortical sites were mapped with unprimed nrTMS and 156 with DCS in all 12 patients. Overall, 33 language-positive areas (17.37\%) were identified during unprimed nrTMS, whereas 30 (19.23\%) were identified during DCS (the rest were motor areas). Although the numbers of detected errors were comparable between the methods, the distribution of these sites did not overlap in most of our patients. Therefore, categorizing these sites into true positive or negative and false positive or negative is not optimal. Instead, we only concentrated on true-positive responses in two patients with minimal cortical distortion. True language-positive areas were considered if the nrTMS and DCS results were within $5 \mathrm{~mm}$ of each other. ${ }^{53}$

The first patient (patient B1) was a 52-year-old man with a tumor located in the left fronto-temporo-insular region (-Table 2). He consulted because of sudden deafness. In this patient, nrTMS stimulation elicited speech arrest from two ROIs (5a and 8a) and phonological errors in one ROI (9a) ( - Fig. 3 left). During DCS, speech arrest was recorded from four ROIs ( $4 b, 5 b, 6 b$, and 7b) ( - Fig. 3 left). Therefore, two true language-positive sites were identified in patient B1 (nrTMS: $5 a$ and 8a; DCS: $5 b$ and $6 b$ ). The rest of the targets

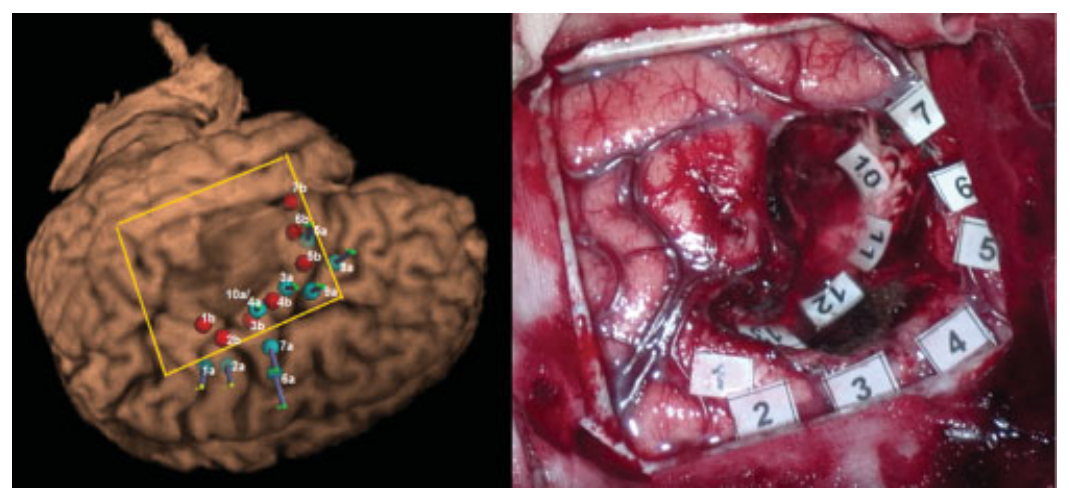

Fig. 3 Comparison of navigated repetitive transcranial magnetic stimulation (nrTMS) and direct cortical stimulation (DCS) mappings in patient B1. Left: Three-dimensional reconstruction of the patient's brain with coregistered nrTMS (cyan landmarks and lowercase "a") and intraoperative DCS (red landmarks and lowercase "b") targets stimulated during picture-naming task. The yellow box marks the area pictured on the right. Right: Intraoperative photograph with numbered positive DCS mapping sites. 


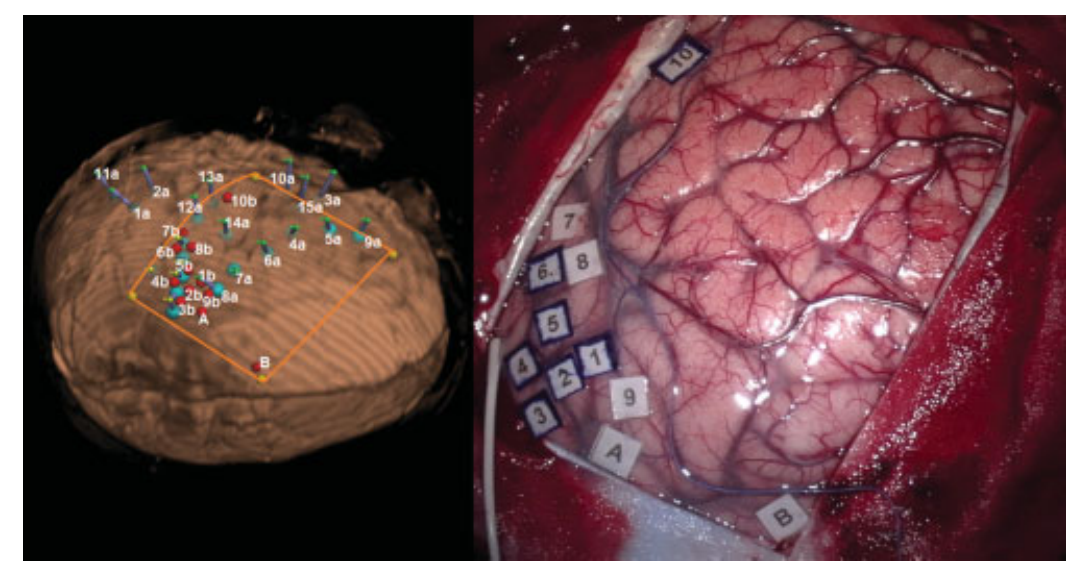

Fig. 4 Comparison of navigated repetitive transcranial magnetic stimulation (nrTMS) and direct cortical stimulation (DCS) mappings in patient B12. Left: Three-dimensional reconstruction of the patient's brain with coregistered nrTMS (cyan landmarks and lowercase "a") and intraoperative DCS (red landmarks and lowercase "b") targets stimulated during picture-naming task. The orange box marks the area pictured on the right. Right: Intraoperative photograph with numbered positive DCS mapping sites (outlined numbers are the common sites for both languages [bilingual patient]).

were not categorized because they did not overlap within $5 \mathrm{~mm}$.

The second patient (patient B12) was a 31-year-old woman with a tumor in the left frontal lobe. She consulted because of new-onset seizures. In this patient, nrTMS was able to elicit speech arrest in three ROIs (9a, 10a, and 13a) (-Fig. 4 left). Speech arrest was elicited by DCS on 10 ROIs (1b to $10 \mathrm{~b}$ left). However, we only identified one truepositive language site (nrTMS: 13a; DCS: 10b) in this patient, and the rest were classified as false-negative sites.

\section{Discussion}

The primary goal of the present study was to explore the influence of prior cortical history or activity on the responsiveness to nrTMS during functional language mapping. We tested this experimentally by priming the cortical target sites with a $5-\mathrm{Hz}$ cTBS paradigm before the real mapping. We compared the ERs from sessions with and without priming stimulation. Our findings demonstrated that priming stimulation influenced the cortical area's response to the subsequent nrTMS mapping paradigm. We had hypothesized that primed cortical areas would respond more to the effect of the nrTMS mapping paradigm, resulting in the detection of more language errors. In contrast, we observed lower ERs during a primed mapping session compared with a session without priming.

\section{Unprimed nrTMS Language Mapping}

In the unprimed nrTMS session, we were able to elicit both minor and major language errors. These results were consistent with previous findings because nrTMS can disrupt language processing and can therefore be used to identify language-eloquent areas in tumor patients. ${ }^{1,10,54}$ The exact underlying mechanism behind the effect of nrTMS paradigms such as cTBS is not yet completely understood. Evidence from motor cortex studies suggests that inhibitory $\gamma-$ aminobutyric acid (GABA) activity may be a mechanism by which long-lasting aftereffects of cTBS on corticospinal excitability are generated. This is because cTBS applied at a low intensity ( $80 \%$ AMT), which is below the threshold for activating the excitatory inputs to pyramidal neurons, was shown to increase the local concentration of GABA in the motor cortex. ${ }^{55,56}$

However, compared with the motor cortex where the aftereffects of cTBS are commonly assessed using MEPs, the cTBS effect on language processing is less clear. This is because language processing is supported by different regions located in separate parts of the brain that need information transfer between them for these regions to function as a network. ${ }^{57}$ In theory, a similar mechanism (localized increase in GABA concentration) could also lead to a decrease in neuronal excitability in specific areas of the language network and produce distinct language errors.

For instance, inhibition of areas such as the left posterior superior temporal gyrus, supramarginal gyrus, and middle temporal gyrus could produce a speech production impairment called phonemic paraphasia or phonological paraphasia (minor error) in our error classification (e.g., a target word such as "pants" is replaced with "plants"). This is because inhibition of these areas partially disrupts the phonological retrieval process, so the phonemes of the spoken word are chosen incorrectly or are incorrectly ordered. ${ }^{58}$ In contrast, inhibition of the Broca's area could produce speech arrest (major error) because this region of the language network accesses the phonological word representation that is compiled sequentially into segments of syllables (i.e., syllabification) and forward it to motor regions where it is transformed into an articulatory (i.e., phonetic) code. $^{59}$

In tumor patients, however, the site-specific response during the stimulation was not consistent with this view. For instance, cTBS stimulation of the frontal lobe elicited phonological paraphasia in some of our patients with a temporoparietal tumor. This is plausible because language processing involves large-scale cortical networks, and tumors could induce a compensatory functional shift of language processes to other areas within the language 
network. As documented in the motor cortex, low- and highgrade gliomas could shift the functional site $>10 \mathrm{~mm}$ to neighboring areas. ${ }^{60}$ Furthermore, tumor-related factors could also affect the responsiveness of cortical neurons to rTMS or even DCS.

Indeed, although we observed comparable numbers of major and minor errors during unprimed nrTMS mapping, the overall ER was low when compared with previous results. This finding could be accounted for by our small sample size. However, we cannot rule out the possibility that large and long-standing gliomas may have already triggered compensatory neuroplasticity (e.g., a shift of language dominance to the right hemisphere) before cortical mapping, thereby causing the nrTMS functional mapping to miss the eloquent site. ${ }^{61}$ Another factor is tumor relapse, the case in two of our patients. It was documented that areas identified as crucial for speech during the first operation could be identified as no longer critical in the second operation (or vice versa) because of compensatory plasticity mechanisms during tumor growth. ${ }^{39,62,63}$

The response to cTBS during language mapping also varied from patient to patient. Multiple errors were elicited in some patients and a few in others. This observation is not surprising, particularly when considering the interindividual variability in CTBS response reported among healthy individuals. ${ }^{64,65}$ Studies showed that variability in cTBS response is also influenced by a common polymorphism in the brainderived neurotrophic factor gene $(B D N F)$. Specifically, individuals who are carriers of the "Val66Met" genotype have reduced cTBS-induced suppression in the motor cortex. ${ }^{66,67}$ Although the correlation of BDNF polymorphism and the response to cTBS during language mapping is unknown, we cannot rule out the possibility that the interpatient response variability was additionally modulated by the BDNF polymorphism. So far, genetic analyses have not been conducted by previous nrTMS studies in brain tumor patients.

\section{Primed nrTMS Language Mapping}

In the primed nrTMS session, we were able to detect significantly lower overall ERs compared with the primed session. In other words, the cortical state induced by the priming stimulation decreased the effectiveness of the actual mapping stimulation. This finding was unexpected because priming stimulations were shown to have a strong modulatory effect (e.g., increased inhibition effect) on motor cortex behavior and language in healthy subjects. ${ }^{34,35,68}$ Originally, we expected to elicit more errors during the primed session, but this was not the case. The absence of the enhancing effect of our priming stimulation might be explained by a homeostatic mechanism that underlies metaplasticity. A homeostatic form of plasticity will act to stabilize the activity of a neuron or neuronal circuit in the face of perturbations. ${ }^{69}$

In our experimental paradigm, we preconditioned the mapping stimulation with cTBS that can also induce inhibition or LTD. Therefore, the induction of LTD during the actual nrTMS mapping would be difficult because the homeostatic mechanism will ensure that one type of synaptic plasticity (e. g., too much LTD) does not predominate the system. ${ }^{70}$ But , if a priming stimulation via a homeostatic mechanism could function as a negative feedback loop to promote a "set point" of synaptic activity (which in theory is variable in tumor patients) within a functional range, ${ }^{70}$ then why did we not achieve better inhibition during the mapping and detect more language errors in our patients?

This question could be answered by the sliding threshold mechanism postulated by the Bienenstock-Cooper-Munro theory of bidirectional synaptic plasticity. According to this theory, a recent postsynaptic firing (excitation) would elevate the threshold for future LTP induction while lowering the threshold for future LTD induction, whereas diminished postsynaptic firing would result in the opposite finding. ${ }^{70-72}$ The scenario in our study would have been the latter because our priming paradigm will diminish (inhibition) postsynaptic firing and therefore elevate the threshold for future LTD. This could have been another reason why the primed stimulation did not work as efficiently as the unprimed stimulation. Future studies using priming should explore the impact of an excitability enhancing rTMS paradigm or the use of transcranial direct current stimulation (tDCS) to prime the mapping stimulation.

Another interesting observation from the primed session were the differences in the ER of minor and major errors. We were able to detect fewer speech arrests and hesitations (major errors) compared with minor errors in the same session and compared with major errors in the unprimed session. Although this effect was relatively modest, it was a clear demonstration that the priming stimulation affected the detection of major errors because they were robustly elicited during the unprimed session. We suggest that tumor location can play a role here. In 10 patients enrolled in the study, the tumor was located in the frontotemporal region close to Broca's area. Indeed, nrTMS mapping over this region produced errors during overt word production such as speech arrests and hesitations (major errors). Broca's area plays a significant role in formulating an appropriate articulatory code that has to be implemented by the motor cortex. $^{59}$ Moreover, it could be argued that verbal output impairments were also congruent with the stimulation of the facial portion of the primary motor cortex in which the stimulation will primarily impair the articulators themselves. ${ }^{59,73}$ However, the latter scenario can be ruled out because measurements with an accompanying strong facial muscle response were excluded from the analysis.

\section{Comparison of Unprimed nrTMS and DCS Language Maps}

Compared with previous nrTMS language mapping studies, we did not adopt an extensive anatomical cortical parcellation system (CPS) because of tumor-induced distortions of cortical areas in most of our patients. This is because different tumor sizes caused different degrees of cortical distortions, making the alignment of each patient data to a common reference space (parcellated cortex) difficult and the interpretations/identification of language-eloquent sites less precise. Therefore, we focus in our study on functionally and anatomically significant areas with respect to the tumor 
location. Our results revealed a low number of overlapping sites or true-positive responses.

The discrepancy in the results of the two mapping methods can be due to several factors. The main factor would be the marked gyri distortions. Growing tumors and the resulting gyri distortions could compromise the accuracy of fMRI in mapping brain function because of abundant abnormal neovasculature. Evidence indicates that abnormal neovasculature decreased the fMRI contrast between oxyhemoglobin and deoxyhemoglobin, leading to a muting of the BOLD signal. $^{74,75}$ The language-specific ROIs only accounts for $59.47 \%$ of the total targets (190). We can therefore not rule out the possibility that language-eloquent areas were not identified as targets because of a weak or absent BOLD response during the language task. Indeed, additional language errors were elicited during the stimulation of the other ROIs recruited by tongue movements and those that were anatomically selected ( $\mathbf{- T a b l e ~} \mathbf{1}$ ).

Brain shift during surgery is another factor that could have affected the result because it was not explicitly measured and corrected in our study. Although we adopted strict guidelines for identifying true-positive sites (within $5 \mathrm{~mm}$ ), brain shift could still account for the lack of true overlap between nrTMS and DCS sites in all the patients we tested. It could be speculated that without brain shift, the true-positive sites we observed from two patients would overlap exactly because stimulation can induce an action potential in adjacent neurons extending millimeters beyond a focal area. ${ }^{76}$ For CPS, a brain shift during surgery could change parcellation boundaries by releasing the anatomical pressure on gyri moving one (nrTMS or DCS) languagepositive site into another region. This could change the category of the site from true positive to false negative because both sites now occupy different anatomical regions. However, it has no deep impact on a real or very close overlap $(\leq 5 \mathrm{~mm}$ ) because both sites could move into a new anatomical region and could still be considered language positive. This issue may be solved by adopting a patient-specific parcellation system that accurately depicts the spatial organization of functional language areas in individual patients.

Additionally, the impact of brain shift in the identification of true-positive sites in our patients could have been influenced by the neuronavigation technique we used during preoperative language mapping. In the study, we utilized a "line-navigated" TMS technique that visualizes the presumed location of the stimulation based on the geometric center of the stimulation coil. This technique differs from an "electric-field navigated" TMS based on the calculation of the electric field generated by the coil and its maximum field strength on the cortex. ${ }^{40}$ Therefore, the language-positive sites detected and marked using the line-navigated TMS technique may not be identical to the location of the maximum electric field responsible for the observable behavioral effect (e.g., speech arrest).

In contrast, electric-field navigated TMS seems favorable in detecting language-positive sites with high concordance to the DCS findings because both stimulations are localized on sites of maximum electric field amplitude (e.g., under- neath the stimulating electrode in DCS). Indeed, data from motor mapping in healthy volunteers and brain tumor patients suggest that line-navigated TMS leads to more limited maps of motor-positive spots. ${ }^{40,77}$ However, language mapping conducted with line and electric-field navigated TMS that compared the results with DCS has not yet been investigated. Therefore, we considered the absence of information concerning the maximum induced electric field a potential limitation of the present study. Future studies must use real-time brain shift corrections, particularly for studies that use a line-navigated TMS technique, to further improve the coregistration and guidance of the DCS probe during stimulation. ${ }^{78}$

\section{Conclusion}

The present pilot study showed for the first time that previous cortical stimulation history has a profound impact on the nrTMS language mapping results in tumor patients. Our findings specifically demonstrated that metaplasticity, as demonstrated using priming stimulation, could still be induced in tumor-affected cortical areas. However, this approach must still be refined to increase the effectiveness of nrTMS in detecting language errors.

\section{Conflict of Interest}

None declared.

\section{References}

1 Picht T, Krieg SM, Sollmann N, et al. A comparison of language mapping by preoperative navigated transcranial magnetic stimulation and direct cortical stimulation during awake surgery. Neurosurgery 2013;72(05):808-819

2 Hervey-Jumper SL, Berger MS. Maximizing safe resection of lowand high-grade glioma. JNeurooncol 2016;130(02):269-282

3 Sollmann N, Picht T, Mäkelä JP, Meyer B, Ringel F, Krieg SM. Navigated transcranial magnetic stimulation for preoperative language mapping in a patient with a left frontoopercular glioblastoma. J Neurosurg 2013;118(01):175-179

4 Gasser T, Ganslandt O, Sandalcioglu E, Stolke D, Fahlbusch R, Nimsky C. Intraoperative functional MRI: implementation and preliminary experience. Neuroimage 2005;26(03):685-693

5 Forster M-T, Hattingen E, Senft C, Gasser T, Seifert V, Szelényi A. Navigated transcranial magnetic stimulation and functional magnetic resonance imaging: advanced adjuncts in preoperative planning for central region tumors. Neurosurgery 2011;68(05): 1317-1324; discussion 1324-1325

6 Roux FE, Boulanouar K, Lotterie JA, Mejdoubi M, LeSage JP, Berry I. Language functional magnetic resonance imaging in preoperative assessment of language areas: correlation with direct cortical stimulation. Neurosurgery 2003;52(06):1335-1345; discussion 1345-1347

7 Zandbelt BB, Gladwin TE, Raemaekers M, et al. Within-subject variation in BOLD-fMRI signal changes across repeated measurements: quantification and implications for sample size. Neuroimage 2008;42(01):196-206

8 Giussani C, Roux FE, Ojemann J, Sganzerla EP, Pirillo D, Papagno C. Is preoperative functional magnetic resonance imaging reliable for language areas mapping in brain tumor surgery? Review of language functional magnetic resonance imaging and direct cortical stimulation correlation studies. Neurosurgery 2010;66 (01):113-120 
9 Krieg SM, Lioumis P, Mäkelä JP, et al. Protocol for motor and language mapping by navigated TMS in patients and healthy volunteers; workshop report. Acta Neurochir (Wien) 2017;159 (07):1187-1195

10 Shamov T, Spiriev T, Tzvetanov P, Petkov A. The combination of neuronavigation with transcranial magnetic stimulation for treatment of opercular gliomas of the dominant brain hemisphere. Clin Neurol Neurosurg 2010;112(08):672-677

11 Hendrix P, Senger S, Simgen A, Griessenauer CJ, Oertel J. Preoperative rTMS language mapping in speech-eloquent brain lesions resected under general anesthesia: a pair-matched cohort study. World Neurosurg 2017;100:425-433

12 Barker AT, Jalinous R, Freeston IL. Non-invasive magnetic stimulation of human motor cortex. Lancet 1985;1(8437):1106-1107

13 Pascual-Leone A, Valls-Solé J, Wassermann EM, Hallett M. Responses to rapid-rate transcranial magnetic stimulation of the human motor cortex. Brain 1994;117(Pt 4):847-85

14 Houdayer E, Degardin A, Cassim F, Bocquillon P, Derambure P, Devanne $H$. The effects of low- and high-frequency repetitive TMS on the input/output properties of the human corticospinal pathway. Exp Brain Res 2008;187(02):207-217

15 Wassermann EM, Wedegaertner FR, Ziemann U, George MS, Chen R. Crossed reduction of human motor cortex excitability by $1-\mathrm{Hz}$ transcranial magnetic stimulation. Neurosci Lett 1998;250(03): 141-144

16 Huang Y-Z, Edwards MJ, Rounis E, Bhatia KP, Rothwell JC. Theta burst stimulation of the human motor cortex. Neuron 2005;45 (02):201-206

17 Hoogendam JM, Ramakers GMJ, Di Lazzaro V. Physiology of repetitive transcranial magnetic stimulation of the human brain. Brain Stimul 2010;3(02):95-118

18 Pascual-Leone A, Walsh V, Rothwell J. Transcranial magnetic stimulation in cognitive neuroscience-virtual lesion, chronometry, and functional connectivity. Curr Opin Neurobiol 2000;10 (02):232-237

19 Kubben PL. Brain mapping: from neural basis of cognition to surgical applications. Surg Neurol Int 2012;3:77

20 Shapiro KA, Pascual-Leone A, Mottaghy FM, Gangitano M, Caramazza A. Grammatical distinctions in the left frontal cortex. J Cogn Neurosci 2001;13(06):713-720

21 Pascual-Leone A, Gates JR, Dhuna A. Induction of speech arrest and counting errors with rapid-rate transcranial magnetic stimulation. Neurology 1991;41(05):697-702

22 Whitney C, Kirk M, O'Sullivan J, Lambon Ralph MA, Jefferies E. The neural organization of semantic control: TMS evidence for a distributed network in left inferior frontal and posterior middle temporal gyrus. Cereb Cortex 2011;21(05):1066-1075

23 Sakai KL, Noguchi Y, Takeuchi T, Watanabe E. Selective priming of syntactic processing by event-related transcranial magnetic stimulation of Broca's area. Neuron 2002;35(06):1177-1182

24 Cappa SF, Sandrini M, Rossini PM, Sosta K, Miniussi C. The role of the left frontal lobe in action naming: rTMS evidence. Neurology 2002; 59(05):720-723

25 Lioumis P, Zhdanov A, Mäkelä N, et al. A novel approach for documenting naming errors induced by navigated transcranial magnetic stimulation. JNeurosci Methods 2012;204(02): 349-354

26 Krieg SM, Tarapore PE, Picht T, et al. Optimal timing of pulse onset for language mapping with navigated repetitive transcranial magnetic stimulation. Neuroimage 2014;100:219-236

27 Hauck T, Tanigawa N, Probst M, et al. Stimulation frequency determines the distribution of language positive cortical regions during navigated transcranial magnetic brain stimulation. BMC Neurosci 2015;16(01):5

28 Hauck T, Tanigawa N, Probst M, et al. Task type affects location of language-positive cortical regions by repetitive navigated transcranial magnetic stimulation mapping. PLoS One 2015;10(04): e0125298
29 Sollmann N, Fuss-Ruppenthal S, Zimmer C, Meyer B, Krieg SM. Investigating stimulation protocols for language mapping by repetitive navigated transcranial magnetic stimulation. Front Behav Neurosci 2018;12(197):197

30 Ille S, Kulchytska N, Sollmann N, et al. Hemispheric language dominance measured by repetitive navigated transcranial magnetic stimulation and postoperative course of language function in brain tumor patients. Neuropsychologia 2016;91:50-60

31 Abraham WC. Metaplasticity: tuning synapses and networks for plasticity. Nat Rev Neurosci 2008;9(05):387

32 Abraham WC, Bear MF. Metaplasticity: the plasticity of synaptic plasticity. Trends Neurosci 1996;19(04):126-130

33 Silvanto J, Bona S, Marelli M, Cattaneo Z. On the mechanisms of transcranial magnetic stimulation (TMS): how brain state and baseline performance level determine behavioral effects of TMS. Front Psychol 2018;9:741

34 Andoh J, Artiges E, Pallier C, et al. Priming frequencies of transcranial magnetic stimulation over Wernicke's area modulate word detection. Cereb Cortex 2008;18(01):210-216

35 Iyer MB, Schleper N, Wassermann EM. Priming stimulation enhances the depressant effect of low-frequency repetitive transcranial magnetic stimulation. JNeurosci 2003;23(34): 10867-10872

36 Kakuda W, Abo M, Momosaki R, Morooka A. Therapeutic application of 6-Hz-primed low-frequency rTMS combined with intensive speech therapy for post-stroke aphasia. Brain Inj 2011;25 (12):1242-1248

37 Oldfield RC. The assessment and analysis of handedness: the Edinburgh inventory. Neuropsychologia 1971;9(01):97-113

38 Rossi S, Hallett M, Rossini PM, Pascual-Leone A; Safety of TMS Consensus Group. Safety, ethical considerations, and application guidelines for the use of transcranial magnetic stimulation in clinical practice and research. Clin Neurophysiol 2009;120(12): 2008-2039

39 Jenkinson M, Beckmann CF, Behrens TEJ, Woolrich MW, Smith SM. FSL. Neuroimage 2012;62(02):782-790. Doi: 10.1016/j. neuroimage.2011.09.015

40 Sollmann N, Goblirsch-Kolb MF, Ille S, et al. Comparison between electric-field-navigated and line-navigated TMS for cortical motor mapping in patients with brain tumors. Acta Neurochir (Wien) 2016;158(12):2277-2289

41 Kilbride RD. Intraoperative functional cortical mapping of language. JClin Neurophysiol 2013;30(06):591-596

42 Nicholas LE, Brookshire RH, Maclennan DL, Schumacher JG, Porrazzo SA. Revised administration and scoring procedures for the Boston Naming test and norms for non-brain-damaged adults. Aphasiology 1989;3(06):569-580

43 Rossini PM, Berardelli A, Deuschl G, et al; The International Federation of Clinical Neurophysiology. Applications of magnetic cortical stimulation. Electroencephalogr Clin Neurophysiol Suppl 1999;52:171-185

44 Saarinen T, Laaksonen H, Parviainen T, Salmelin R. Motor cortex dynamics in visuomotor production of speech and non-speech mouth movements. Cereb Cortex 2006;16(02):212-222

45 Ille S, Sollmann N, Hauck T, et al. Combined noninvasive language mapping by navigated transcranial magnetic stimulation and functional MRI and its comparison with direct cortical stimulation. J Neurosurg 2015;123(01):212-225

46 Corina DP, Loudermilk BC, Detwiler L, Martin RF, Brinkley JF, Ojemann G. Analysis of naming errors during cortical stimulation mapping: implications for models of language representation. Brain Lang 2010;115(02):101-112

47 Oberman L, Edwards D, Eldaief M, Pascual-Leone A. Safety of theta burst transcranial magnetic stimulation: a systematic review of the literature. JClin Neurophysiol 2011;28(01):67-74

48 Wassermann EM. Risk and safety of repetitive transcranial magnetic stimulation: report and suggested guidelines from the International Workshop on the Safety of Repetitive Transcranial 
Magnetic Stimulation, June 5-7, 1996. Electroencephalogr Clin Neurophysiol 1998;108(01):1-16

49 Sanai N, Mirzadeh Z, Berger MS. Functional outcome after language mapping for glioma resection. N Engl J Med 2008;358(01): $18-27$

50 Ojemann G, Ojemann J, Lettich E, Berger M. Cortical language localization in left, dominant hemisphere. An electrical stimulation mapping investigation in 117 patients. J Neurosurg 1989;71 (03):316-326

51 Tarapore PE, Findlay AM, Honma SM, et al. Language mapping with navigated repetitive TMS: proof of technique and validation. Neuroimage 2013;82:260-272

52 Kong NW, Gibb WR, Tate MC. Neuroplasticity: insights from patients harboring gliomas. Neural Plast 2016;2016:2365063

53 Lehtinen H, Mäkelä JP, Mäkelä T, et al. Language mapping with navigated transcranial magnetic stimulation in pediatric and adult patients undergoing epilepsy surgery: Comparison with extraoperative direct cortical stimulation. Epilepsia Open 2018;3 (02):224-235

54 Rösler J, Niraula B, Strack V, et al. Language mapping in healthy volunteers and brain tumor patients with a novel navigated TMS system: evidence of tumor-induced plasticity. Clin Neurophysiol 2014;125(03):526-536

55 Alle $\mathrm{H}$, Heidegger T, Kriváneková L, Ziemann U. Interactions between short-interval intracortical inhibition and short-latency afferent inhibition in human motor cortex. J Physiol 2009;587(Pt 21):5163-5176

56 Stagg CJ, Wylezinska M, Matthews PM, et al. Neurochemical effects of theta burst stimulation as assessed by magnetic resonance spectroscopy. J Neurophysiol 2009;101(06):2872-2877

57 Friederici AD, Gierhan SME. The language network. Curr Opin Neurobiol 2013;23(02):250-254

58 Binder JR. The Wernicke area: modern evidence and a reinterpretation. Neurology 2015;85(24):2170-2175

59 Flinker A, Korzeniewska A, Shestyuk AY, et al. Redefining the role of Broca's area in speech. Proc Natl Acad Sci U S A 2015;112(09): 2871-2875

60 Thiel A, Habedank B, Herholz K, et al. From the left to the right: how the brain compensates progressive loss of language function. Brain Lang 2006;98(01):57-65

61 Devlin JT, Watkins KE. Stimulating language: insights from TMS. Brain 2007;130(Pt 3):610-622

62 Conway N, Wildschuetz N, Moser T, et al. Cortical plasticity of motor-eloquent areas measured by navigated transcranial magnetic stimulation in patients with glioma. J Neurosurg 2017;127 (05):981-991

63 Sarubbo S, Le Bars E, Moritz-Gasser S, Duffau H. Complete recovery after surgical resection of left Wernicke's area in awake patient: a brain stimulation and functional MRI study. Neurosurg Rev 2012;35(02):287-292; discussion 292
64 Lowe CJ, Hall PA. Reproducibility and sources of interindividual variability in the responsiveness to prefrontal continuous theta burst stimulation (cTBS). Neurosci Lett 2018;687:280-284

65 Vernet M, Bashir S, Yoo W-K, et al. Reproducibility of the effects of theta burst stimulation on motor cortical plasticity in healthy participants. Clin Neurophysiol 2014;125(02):320-326

66 Cheeran B, Talelli P, Mori F, et al. A common polymorphism in the brain-derived neurotrophic factor gene (BDNF) modulates human cortical plasticity and the response to rTMS. J Physiol 2008;586 (23):5717-5725

67 Jannati A, Block G, Oberman LM, Rotenberg A, Pascual-Leone A. Interindividual variability in response to continuous theta-burst stimulation in healthy adults. Clin Neurophysiol 2017;128(11): 2268-2278

68 Southwell DG, Hervey-Jumper SL, Perry DW, Berger MS. Intraoperative mapping during repeat awake craniotomy reveals the functional plasticity of adult cortex. J Neurosurg 2016;124(05): 1460-1469

69 Hurley R, Machado L. Using tDCS priming to improve brain function: can metaplasticity provide the key to boosting outcomes? Neurosci Biobehav Rev 2017;83:155-159

70 Turrigiano GG. The self-tuning neuron: synaptic scaling of excitatory synapses. Cell 2008;135(03):422-435

71 Cassidy JM, Gillick BT, Carey JR. Priming the brain to capitalize on metaplasticity in stroke rehabilitation. Phys Ther 2014;94(01): 139-150

72 Bienenstock EL, Cooper LN, Munro PW. Theory for the development of neuron selectivity: orientation specificity and binocular interaction in visual cortex. J Neurosci 1982;2(01):32-48

73 Indefrey P. The spatial and temporal signatures of word production components: a critical update. Front Psychol 2011; $2: 255$

74 Fraga de Abreu VH, Peck KK, Petrovich-Brennan NM, Woo KM, Holodny AI. Brain tumors: the influence of tumor type and routine MR imaging characteristics at BOLD functional MR imaging in the primary motor gyrus. Radiology 2016;281(03): 876-88

75 Hou BL, Bradbury M, Peck KK, Petrovich NM, Gutin PH, Holodny AI. Effect of brain tumor neovasculature defined by rCBV on BOLD fMRI activation volume in the primary motor cortex. Neuroimage 2006;32(02):489-497

76 Epstein CM, Meador KJ, Loring DW, et al. Localization and characterization of speech arrest during transcranial magnetic stimulation. Clin Neurophysiol 1999;110(06):1073-1079

77 Sollmann N, Hauck T, Obermüller T, et al. Inter- and intraobserver variability in motor mapping of the hotspot for the abductor policis brevis muscle. BMC Neurosci 2013;14(01):94

78 Histed MH, Bonin V, Reid RC. Direct activation of sparse, distributed populations of cortical neurons by electrical microstimulation. Neuron 2009;63(04):508-522 